\title{
Enhanced Transmission at a Spinal Synapse Triggered In Vivo by an Injury Signal Independent of Altered Synaptic Activity
}

\author{
Edyta K. Bichler, ${ }^{1 *}$ Stan T. Nakanishi, ${ }^{3 *}$ Qing-Bo Wang, ${ }^{2}$ Martin J. Pinter, ${ }^{1}$ Mark M. Rich, ${ }^{2}$ and Timothy C. Cope ${ }^{2}$ \\ 'Department of Physiology, Emory University, Atlanta, Georgia 30345, 2Department of Neuroscience, Cell Biology and Physiology, Wright State University, \\ Dayton, Ohio 45435, and ${ }^{3}$ Hotchkiss Brain Institute, University of Calgary, Calgary, Alberta, Canada T2N 4N1
}

\begin{abstract}
Peripheral nerve crush initiates a robust increase in transmission strength at spinal synapses made by axotomized group IA primary sensory neurons. To study the injury signal that initiates synaptic enhancement in vivo, we designed experiments to manipulate the enlargement of EPSPs produced in spinal motoneurons (MNs) by IA afferents $3 \mathrm{~d}$ after nerve crush in anesthetized adult rats. If nerve crush initiates IA EPSP enlargement as proposed by reducing impulse-evoked transmission in crushed IA afferents, then restoring synaptic activity should eliminate enlargement. Daily electrical stimulation of the nerve proximal to the crush site did, in fact, eliminate enlargement but was, surprisingly, just as effective when the action potentials evoked in crushed afferents were prevented from propagating into the spinal cord. Consistent with its independence from altered synaptic activity, we found that IA EPSP enlargement was also eliminated by colchicine blockade of axon transport in the crushed nerve. Together with the observation that colchicine treatment of intact nerves had no short-term effect on IA EPSPs, we conclude that enhancement of IA-MN transmission is initiated by some yet to be identified positive injury signal generated independent of altered synaptic activity. The results establish a new set of criteria that constrain candidate signaling molecules in vivo to ones that develop quickly at the peripheral injury site, move centrally by axon transport, and initiate enhanced transmission at the central synapses of crushed primary sensory afferents through a mechanism that can be modulated by action potential activity restricted to the axons of crushed afferents.
\end{abstract}

Key words: retrograde; plasticity; motoneurons; sensory neurons; sensorimotor; spinal cord

\section{Introduction}

The full set of mechanisms inducing synaptic adaptation in the adult mammalian CNS remains unknown, especially in vivo. Incomplete understanding is evident when considering changes in transmission at synapses for which the presynaptic neuron is damaged. Within a few days after crush of a muscle nerve, the monosynaptic EPSPs produced in vivo by damaged IA muscle spindle afferents are enlarged by approximately twofold at synapses with uninjured motoneurons (MNs) (Miyata and Yasuda, 1988; Manabe et al., 1989; Seburn and Cope, 1998). In this injury, the peripheral axons (motor and sensory) are severed, but the centrally projecting branches of damaged primary afferents, including IA afferents, remain in continuity both with their cell bodies in the dorsal root ganglion and with the synapses they make with MNs in uninjured spinal motor pools. This axonal injury causes substantial changes in the normal firing behavior of

Received May 2, 2007; revised 0ct. 5, 2007; accepted 0ct. 6, 2007.

This work was supported by National Institute of Neurological Disorders and Stroke Grant R01 NS038693-05 (T.C.C.) and National Research Service Award F31 NS46982 (S.T.N.).

*E.K.B. and S.T.N. contributed equally to this work.

Correspondence should be addressed to Dr. Timothy C. Cope, Department of Neuroscience, Cell Biology and Physiology, Wright State University School of Medicine, 3640 Colonel Glenn Highway, Dayton, OH 45435. E-mail: timothy.cope@wright.edu.

DOI:10.1523/JNEUROSCI.1997-07.2007

Copyright $\odot 2007$ Society for Neuroscience 0270-6474/07/2712851-09\$15.00/0 both IA afferents and MNs. Large-diameter muscle afferents, including IA afferents, express a substantial reduction in action potential (AP) firing (Michaelis et al., 2000), with most afferents (estimated 80\%) falling silent and the remainder firing spontaneously at low average rates $(\sim 2.5 \mathrm{pps})$. MNs that are spared injury express greater than normal activity soon after damage to their functional synergists (Pearson et al., 1999). If these changes in presynaptic or postsynaptic activity are responsible for the injury-induced enhancement at their central synapses, then the cause(s) cannot be attributed to any of the well known mechanisms by which altered neural activity produces a sustained change in synaptic strength. The injury-induced increase in IA-MN synaptic strength does not follow a Hebbian rule, because it is opposite to the reduced strength expected when firing in presynaptic and postsynaptic cells is uncoupled (Stent, 1973). It is also at odds with the homeostatic rule, because synaptic efficacy should be scaled down, not up, by increased MN activity (Turrigiano and Nelson, 1998; Davis, 2006). Finally, injury-induced strengthening at the IA-MN synapse is unlikely to be a form of long-term potentiation (LTP) because frequency-dependent potentiation at mature IA-MN synapses does not persist but fades within minutes (Lev-Tov et al., 1983).

Enhancement after nerve injury may reflect a different kind of activity regulation. Similar to nerve crush, chronic tetrodotoxin blockade of AP conduction in peripheral nerves reduces or elim- 
inates impulse invasion of IA synapses and enlarges EPSPs at IA-MN synapses (Gallego et al., 1979; Manabe et al., 1989; Webb and Cope, 1992). These observations led Kuno (1995) and coworkers to hypothesize that synapses can be strengthened by presynaptic "disuse." To test this hypothesis, we proposed that replacing activity in crushed IA afferents should prevent, or at least reduce, the IA EPSP enlargement. Here we report that daily electrical stimulation of the central end of a crushed nerve did indeed eliminate EPSP enlargement, but through some mechanism not requiring restoration of impulse-evoked activity at central synapses. Our findings suggest instead that the in vivo signaling mechanism for synaptic enhancement involves transport of molecules introduced or activated near the peripheral site of nerve crush.

\section{Materials and Methods}

Studies were performed on 49 adult (260-330 g) female Wistar rats as approved by the Emory University Institutional Animal Care and Use Committee. Rats were anesthetized throughout survival surgery, treatment, and terminal experiments as described below, after which they were killed by barbiturate overdose ( $150 \mathrm{mg} / \mathrm{kg}$ Nembutal, i.p.).

\section{Experimental paradigm}

These studies were designed to open investigation of injury signals that trigger enhanced transmission at IA-MN synapses. The experimental paradigm is illustrated in Figure 1. Stimulus-evoked IA EPSPs in MNs were measured during terminal experiments performed on 10 normal rats (no nerve treatment or crush) and on 39 others within $4 \mathrm{~d}$ of nerve crush and/or nerve treatment as described below.

Nerve crush. Selected portions of the tibial nerve were exposed by skin incision, isolated from surrounding nerves, and crushed by firm pressure applied for $10 \mathrm{~s}$ between the prongs of fine jeweler's forceps. The nerve was deeply indented at the crush site but was not physically severed. Crush was applied either to the tibial nerve at the rostral end of the popliteal fossa or to the nerve supplying the lateral gastrocnemius and soleus (LGS) muscles within $5 \mathrm{~mm}$ of the LG muscle hilus. Failure during the terminal experiment to elicit muscle contraction by electrical stimulation proximal to the crush site verified the completeness of axonal interruption in all cases.

Nerve treatments. All treatments were applied using sterile procedures on rats deeply anesthetized by inhalation of $1-2 \%$ isofluorane in room air. When treatment was complete, skin incisions were closed by wound clips, anesthesia was discontinued, and rats were returned to their cages after full recovery of consciousness.

\section{Daily electrical stimulation}

The effects of daily electrical stimulation were studied in rats with LGS nerve crush. Because selective stimulation of the LGS nerve was not feasible under these conditions (short length and limited spatial access of the LGS nerve), the effects were assessed instead by applying stimulation to the whole tibial nerve, which contains the axons destined for the LGS nerve in the proximal popliteal fossa (see Fig. $3 c$ ). To assess nonspecific effects of daily electrical stimulation in a separate group of rats, the stimulation site was moved distal to the LGS nerve and adjacent the medial gastrocnemius (MG) nerve, thereby excluding those nerves from stimulation while including all other tibial nerve branches (see Fig. 3d). Sterilized bipolar electrodes were positioned on the tibial nerve at either the proximal or distal site for electrical stimulation with brief pulses ( $40 \mu \mathrm{s})$ for a period of $1 \mathrm{~h}$ per day. The optimal stimulus parameters were not known a priori and were guided by the following considerations. First, stimulation frequency was set at $20 \mathrm{~Hz}$, because it is within the range observed by Haftel et al. (2004) for resting discharge of intact spindle afferents in anesthetized rats. Second, stimulation was applied continuously to maximize stimulus pulse number and minimize the total time that the rat was under anesthesia. Finally, stimulus strength was set at twice threshold for producing visible contraction of limb muscles, to preferentially activate neurons with the largest-diameter axons, namely motor axons and group I afferents, and to exclude activation of smaller-

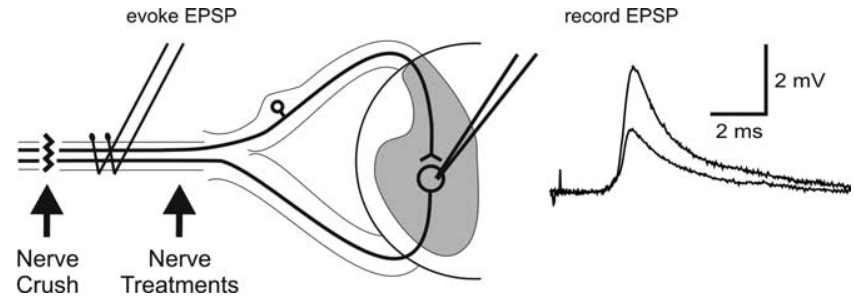

Figure 1. Key elements of experimental paradigm. EPSPs were evoked by electrical stimulation (1 pps, group I strength) and recorded intracellularly from MNs in the spinal cords of anesthetized rats in vivo during terminal experiments as shown in top portion of the figure. Superimposed EPSPs illustrate the average enlargement $(\sim 2 \times)$ produced $3 \mathrm{~d}$ after nerve crush relative to normal. Measurements were made in multiple groups of rats, including rats with intact and untreated nerves (normal) and ones with nerve crush and/or various nerve treatments (details in text)

diameter afferents (e.g., groups III and IV). Stimulation under these conditions was constantly monitored by the experimenter. After $1 \mathrm{~h}$ of stimulation, the electrodes were removed, the wound was closed by wound clips, anesthesia was discontinued, and the rat returned to its cage after full recovery from anesthesia. The number of stimulation sessions (one per day, up to $3 \mathrm{~d}$ ) and the site of stimulation varied as described in Results.

In some rats that underwent daily stimulation, the fast $\mathrm{Na}^{+}$channel blocker lidocaine was applied to the sciatic nerve between the tibial nerve stimulation site and the spinal cord. We determined that the lidocaine procedure described below was effective in blocking the centripetal conduction of APs initiated by daily stimulation (see Fig. 5A), as required to determine whether the effects of stimulation involved activation of spinal synapses. In isofluorane-anesthetized rats, the sciatic nerve was exposed by skin incision near the head of the femur. For 10 min preceding and throughout daily stimulation of the tibial nerve as described above, the sciatic nerve was soaked in a diluted solution of 5\% lidocaine in sterile isotonic saline. On completion of the $1 \mathrm{~h}$ of daily stimulation, the lidocaine solution was removed, and the sciatic nerve was flushed repeatedly with sterile saline before closing wound sites and returning conscious rats to their cages as described above.

\section{Chronic blockade of axon transport}

Axon transport was blocked in some rats to test its role in mediating synaptic enhancement caused by nerve crush. The left tibial nerve was exposed at mid-thigh by skin incision and dissection of the overlying biceps femoris muscle. Connective tissue was gently removed over 2-3 $\mathrm{mm}$ of the tibial nerve where a small piece of gelfoam was applied for 20 min. The gelfoam was soaked in sterile saline solution either with or without $25 \mathrm{~mm}$ colchicine. Application of colchicine in this way blocks axon transport for durations long exceeding the few days preceding the terminal experiments (Jackson and Diamond, 1977).

Several observations suggested that axonal AP activity and conduction was unaffected by colchicine treatment. Repeated behavioral observations made after survival surgery revealed that the treated limb exhibited normal motor behavior (weight support in posture and normal walking and standing on hindlimbs) and normal sensorimotor responses to palpation and gentle manipulation of the foot and leg. When hind quarters were suspended by lifting the tail, no difference was noted in limb extension and toe splaying between the treated and untreated limbs. During the terminal experiment, we consistently found that low stimulus strengths of electrical stimulation caused strong contraction of distal limb muscles when applied proximal to the colchicine treatment site and robust compound APs in dorsal roots and IA EPSPs in MNs (see below) when applied distal to the treatment site. Additionally, colchicine treatment did not prevent injury discharge or its propagation, as evidenced by muscle contraction elicited during nerve crush. These observations suggest that the transient application of colchicine used here did not block AP conduction or impulse-evoked transmission at central synapses.

\section{Terminal experiments}

All rats $(n=49)$ were used in terminal experiments. Pentobarbital (Nembutal) was used to induce $(50 \mathrm{mg} / \mathrm{kg}$, i.p.) and maintain $(3-6 \mathrm{mg} /$ 


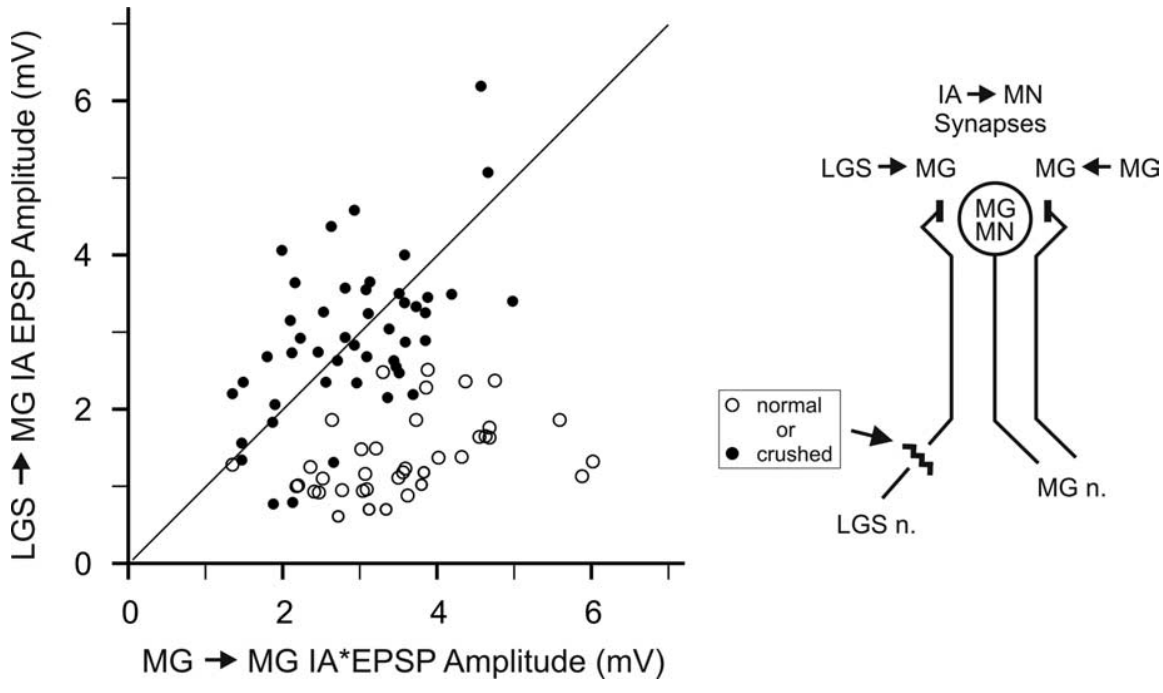

Figure 2. Synaptic enhancement was restricted to IA-MN synapses with presynaptic injury. Right, The diagram shows synaptic input to one MG MN both from MG IA afferents $(M G \rightarrow M G)$ that were uncrushed and from LGS IA afferents (LGS $\rightarrow M G)$ that were either uncrushed (open circles) or crushed (jagged line in diagram) 3 dearlier (filled circles). Left, The graph plots amplitudes of IA EPSPs evoked from LGS versus MG IA afferents in the same individual MNs. IA EPSPs from crushed LGS IA afferents were often larger than those from uncrushed MG IA afferents ( 29 of 47 filled circles above line of identity) but were never larger from uncrushed LGS IA afferents ( 0 of 38 open circles all below the line).

$\mathrm{kg} / \mathrm{h}$, i.p.) anesthesia at a deep level judged from the absence of withdrawal reflexes. Experiments proceeded when vital signs were within acceptable limits: mean carotid blood pressure $>70 \mathrm{mmHg}$, end-tidal $\mathrm{CO}_{2}$ between 3 and $4 \%$, and rectal temperature between 36 and $38^{\circ} \mathrm{C}$. Rats were prepared for electrophysiological recording as described previously (Haftel et al., 2005). Briefly, after laminectomy and dissection of the left hindlimb exposed spinal segments L4-S1 and selected tibial nerve branches, respectively, the rats were secured in a rigid frame at the head, lateral aspects of spinal vertebrae, and left hindlimb for purposes of recording electrophysiological data. At the conclusion of data collection, rats were killed by pentobarbital overdose ( $150 \mathrm{mg} / \mathrm{kg}$, i.p.), and treated nerves were harvested for Western blot analysis.

\section{Data collection}

Spinal MNs penetrated by sharp glass micropipettes (5-10 M $2 ; 2 \mathrm{M}$ $\mathrm{K}$-acetate) were identified as either tibial or MG MNs when antidromic APs were generated by electrical stimulation of the corresponding peripheral nerves. Data collection proceeded when antidromic AP amplitude exceeded $60 \mathrm{mV}$. Rheobase current was measured as the least amount of depolarizing current (50 ms duration square pulse) required to elicit an AP, and APs were evoked by suprathreshold current pulses (0.5 ms duration) for later analysis of afterhyperpolarization (AHP). Next, short-latency EPSPs were evoked by electrical stimulation (Fig. 1) of selected peripheral nerves (pulse duration, $40 \mu \mathrm{s}$; frequency, $0.5 \mathrm{~Hz}$; strength, 2.5 times threshold for compound APs in dorsal roots recorded by bipolar electrodes from dorsal root L5). The peak of the shortestlatency EPSP is dominated by monosynaptic connections between spindle afferents (predominantly group IA) and MNs (Cope et al., 2001) and from this point forward is referred to as the IA EPSP. The electrical stimulus used to evoke IA EPSPs in MNs from homonymous afferents (i.e., IA EPSPs generated in MG MNs by stimulating the MG nerve or in tibial MNs by stimulating the tibial nerve) often generated antidromic APs in MNs that obscured the IA EPSPs. To reveal the peak of the IA EPSP, hyperpolarizing current was injected into the $\mathrm{MN}$ as required to block the antidromic AP (Manabe et al. 1989). Records of MN membrane potential, electrode current, and compound APs in dorsal roots were digitized and stored on computer for off-line analysis.

\section{Data analysis}

Voltage traces of IA EPSPs and APs generated by current injection were averaged from replicate stimulus trials $(\sim 10)$, to improve measurement accuracy by enhancing the signal-to-noise ratio. Peak amplitude was measured from the averaged IA EPSPs, and the time taken for AHP to decay from peak hyperpolarization to half-amplitude (AHP halfdecay time) was measured from the currentevoked APs. Nested ANOVAs were used to test for treatment effects of IA EPSP amplitudes and MN electrical properties, and Tukey's honestly significant difference (HSD) post hoc tests were used to test for the significance of comparisons of treatment effects using commercial software (SYSTAT; Systat Software, Point Richmond, CA). Data are reported as mean \pm SEM unless indicated otherwise.

The effectiveness of colchicine in blocking axon transport was assessed by harvesting the tibial nerves from colchicine-treated rats at the end of the terminal experiments. Western blot analysis was used to establish that the vesicular protein SV2 accumulated in the tibial nerve as expected if colchicine blocked axon transport (Li et al., 1999). Tibial nerves were removed, quickly frozen in liquid nitrogen, and stored at $-80^{\circ} \mathrm{C}$. The frozen tissue was minced, homogenized separately in ice-cooled T-PEP tissue protein extraction reagent (Pierce, Rockford, IL) containing a protease inhibitor mixture (Roche, Basel, Switzerland), and centrifuged for $10 \mathrm{~min}$ at $10,000 \times \mathrm{g}$. The clear supernatant was withdrawn and assayed for protein content with a BCA kit (Pierce) Proteins $(30 \mu \mathrm{g})$ were mixed with Laemmli sample buffer, heated for 5 min at $70^{\circ} \mathrm{C}$, electrophoresed in precast $7.5 \%$ gels (Bio-Rad, Hercules, $\mathrm{CA}$ ), and transferred to polyvinylidene difluoride membranes (Millipore, Bedford, MA). After blocking for $1 \mathrm{~h}$ in PBS containing $5 \%$ nonfat dry milk and $0.1 \%$ Tween 20 , the membranes were first incubated with mouse monoclonal anti-SV2 antibody (1:200; Developmental Studies Hybridoma Bank, Iowa City, IA), followed by a second incubation with horseradish peroxidase-conjugated anti-mouse IgG antibody (1:4000; Chemicon, Temecula, CA). The blots were visualized using ECL detection reagents (Amersham Biosciences, Piscataway, NJ) on Kodak (Rochester, NY) film. To control for protein loading, the same membranes were stripped, reprobed with affinity-purified polyclonal anti-actin antibody (1:1000; Santa Cruz Biotechnology, Santa Cruz, CA). For a negative control, blots were incubated in a secondary antibody only. The intensity of bands in gels was quantified using SigmaScan software.

\section{Results}

Synaptic enhancement depends on presynaptic injury

IA EPSPs more than doubled in size $3 \mathrm{~d}$ after crushing either the LGS nerve or the tibial nerve (Miyata and Yasuda, 1988; Manabe et al., 1989; Seburn and Cope, 1998). Before detailing the effects of nerve crush and the conditions that modify them, we report new information on the selectivity of EPSP enlargement. In some cases, it was possible to record the IA EPSPs elicited from two different sources of IA input onto the same MG MNs (Fig. 2, diagram). For normal rats, the plot in Figure 2 verifies previous demonstrations (Burke et al., 1978; Manabe et al., 1989) that IA EPSPs produced at LGS $\rightarrow$ MG (heteronymous) synapses were smaller than those generated at $\mathrm{MG} \rightarrow \mathrm{MG}$ (homonymous) synapses; the average heteronymous/homonymous amplitude ratio was $0.43 \pm 0.03$, and no data points were above the line of identity in Figure 2 ( 0 of 38 open circles). Three days after nerve crush, IA EPSPs produced by crushed LGS afferents were larger than those evoked by normal MG afferents, expressing a significantly larger average amplitude ratio $(1.02 \pm 0.09 ; p<0.001)$ and having 29 of 47 data points (filled circles) exceeding the line of identity. This treatment effect was the result of a selective enlargement of IA EPSPs from crushed afferents, as indicated by the obvious up- 


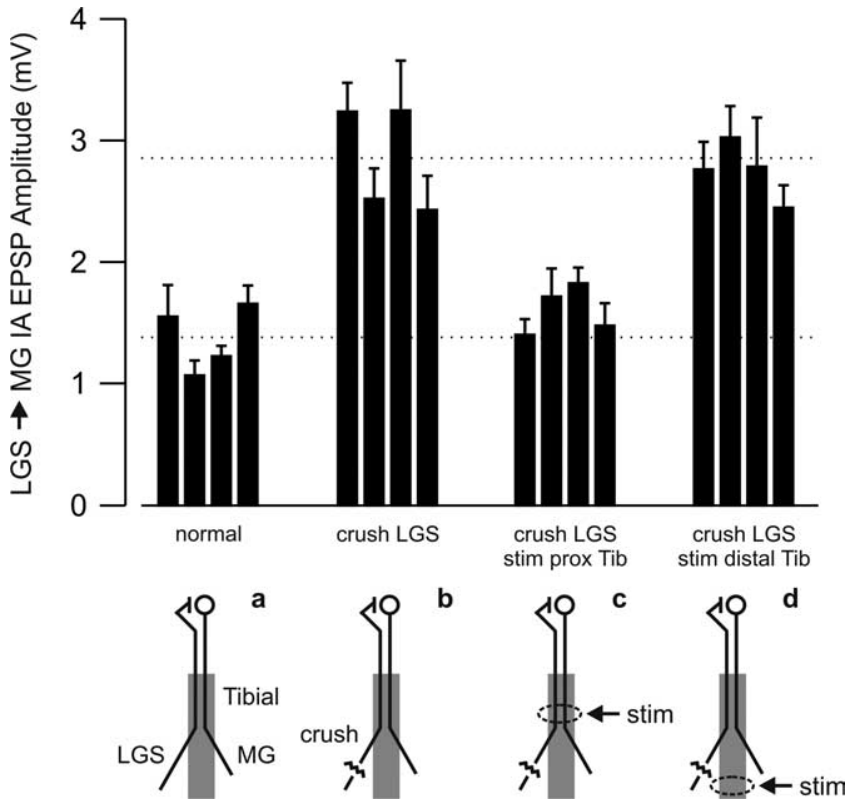

Figure 3. Daily nerve stimulation eliminated injury-induced synaptic enhancement. The bar graph shows mean \pm SEM (bars and brackets, respectively) for amplitude of LGS $\rightarrow$ MG IA EPSPs sampled from each of four rats in four experimental groups. Associated diagrams depict the IA-MN synapse formed by an LGS IA afferent and an MG MN [afferent and efferent axons projecting as black lines through the gray bar, which represents the tibial nerve (Tib) and all of the axons that project through it]. Groups from left to right are as follows: $\boldsymbol{a}$, normal; $\boldsymbol{b}, 3 \mathrm{~d}$ LGS nerve crush (jagged line in diagram); $3 \mathrm{~d}$ LGS nerve crush with daily stimulation (stim) of the tibial nerve at sites (dashed ovals and arrows) that either included (proximal) (c) or excluded (distal) (d) the crushed LGS and intact MG nerves. Electrical stimulation was applied at group I strength, 20 pps continuously for $1 \mathrm{~h} / \mathrm{d}$. Top and bottom horizontal dotted lines in the graph represent, respectively, the mean of mean values for LGS $\rightarrow$ MG IA EPSP amplitudes from rats with LGS nerve either crushed or normal. The graph shows that proximal but not distal stimulation prevented crush-induced IA EPSP enlargement.

ward shift on the $y$-axis for data from nerve crushed rats relative to normal rats but no apparent shift on the $x$-axis. Enlargement of IA EPSPs was restricted, therefore, to synapses made by presynaptic neurons having crushed peripheral axons (LGS $\rightarrow$ MG) and was not expressed at neighboring synapses made with the same $\mathrm{MNs}$ by uncrushed IA afferents $(\mathrm{MG} \rightarrow \mathrm{MG})$.

\section{Activity modulates crush-induced synaptic enhancement}

We hypothesized that if nerve crush caused IA EPSP enlargement by reducing presynaptic firing and associated synaptic transmission (see Introduction), then restoring impulse-evoked transmission should prevent enlargement. To test this hypothesis, APs were evoked in the tibial nerve by daily electrical stimulation (see Materials and Methods). The results are illustrated in Figure 3 as means for individual animals (Table 1 presents group mean of mean values and statistical comparisons). The increase in IA EPSP amplitude caused by nerve crush (Fig. $3 b$ ) was eliminated when daily stimulation $(20 \mathrm{~Hz}$ for $1 \mathrm{~h}$; see Materials and Methods) was applied proximally on the tibial nerve at a site that included the LGS nerve above the crush site and included the intact MG nerve (Fig. 3c). In contrast, stimulation was ineffective when applied distally on the tibial nerve at a site that excluded any portion of the MG or LGS nerves (Fig. 3d). Thus, elimination of IA EPSP enlargement necessitated stimulation of the crushed LGS nerve in particular and was not attributable more generally to nonspecific actions of electrical stimulation on, for example, other tibial afferents, MNs, or spinal circuits.

The possible effects of electrical stimulation on IA-MN syn-

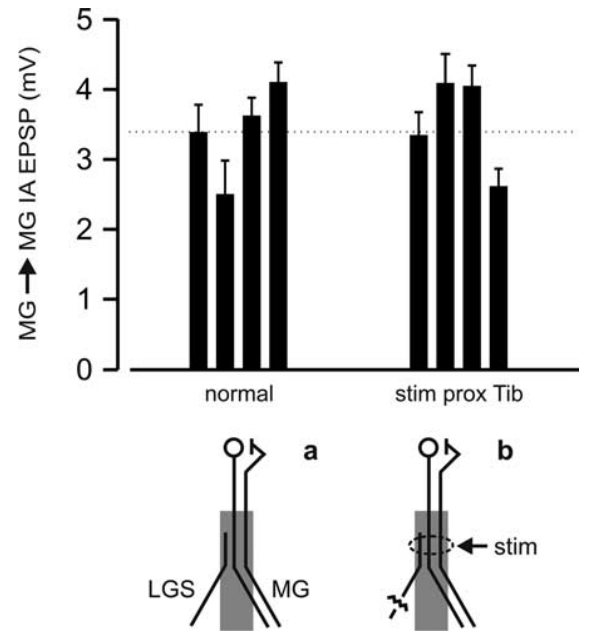

Figure 4. Daily stimulation had no effect on synaptic function of uncrushed IA afferents. The bar graph shows mean \pm SEM for MG $\rightarrow$ MG IA EPSP amplitudes from each of four rats in two groups. Associated diagrams (compare Fig. 3) identify MG $\rightarrow$ MG IA-MN synapses in normal rats $(\boldsymbol{a})$ and in rats $(\boldsymbol{b})$ with crushed LGS nerve and daily stimulation of proximal tibial nerve (stim prox tib; dotted oval and arrow) that included the MG nerve. The dotted horizontal line shows mean of means at MG $\rightarrow$ MG IA-MN synapses for normal rats ( $3.40 \pm 0.34 \mathrm{mV} ; n=38$ ), which was not significantly different than that for rats with LGS nerve crush and daily tibial nerve stimulation (3.52 $\pm 0.35 \mathrm{mV} ; n=28 ; p=0.83$ ).

aptic function (i.e., effects distinct from activating the crushed nerve) were assessed by examining EPSPs at MG $\rightarrow$ MG synapses. At these synapses, both presynaptic and postsynaptic elements (IA afferents and MNs) were included in proximal tibial nerve stimulation but were not crushed, and the MNs were the same ones in which proximal stimulation eliminated crush-induced enlargement of LGS IA EPSPs (Fig. 3c, Table 1). At these $\mathrm{MG} \rightarrow \mathrm{MG}$ synapses, daily electrical stimulation had no significant effect on IA EPSP amplitude (Fig. 4). This finding shows that daily stimulation of uncrushed IA afferents or MNs was not sufficient to alter IA EPSPs. These and findings presented above point to group IA afferents in the crushed LGS nerve as the locus at which daily stimulation prevented IA EPSP enlargement. The ability to eliminate IA EPSP enlargement by restoring activity specifically to crushed primary afferents was consistent with the hypothesis that nerve crush enhanced synaptic strength by reducing presynaptic activity.

One attempt was made to identify the parameters of daily stimulation required to prevent IA EPSP enlargement. In this case, daily stimulation of the tibial nerve was restricted to $1 \mathrm{~h}$ on the second day after LGS nerve crush. The mean amplitude of IA EPSPs measured under this condition is reported in Table 1 and was not significantly different from rats with no stimulation. This suggests that manipulation of the IA EPSP enlargement requires daily maintenance of activity in the crushed nerve, or possibly stimulation immediately after nerve crush.

Table 1 reports the group mean of mean values for electrical properties of the MG MNs from which IA EPSPs were recorded. These data enabled assessment of whether a potential bias in MN sampling influenced group means for IA EPSP amplitude. IA EPSP size normally varies across MNs supplying single muscles (Fig. 2), and the postsynaptic contribution to this variance can be estimated by rheobase current and AHP half-decay time, because these MN properties normally covary with IA EPSP size (Kuno, 1995). These and other postsynaptic properties (e.g., input resistance) are not changed in injury-spared MG MNs only $3 \mathrm{~d}$ after crushing the LGS nerve (Miyata and Yasuda, 1988; Seburn and 
Table 1. Nerve treatment effects on LGS $\rightarrow$ MG IA EPSP amplitude and other properties recorded from MG MNs

\begin{tabular}{|c|c|c|c|c|c|}
\hline Treatment & $\begin{array}{l}\text { Number of } \\
\text { rats }\end{array}$ & $\begin{array}{l}\text { Number of MNs } \\
\text { (range MN/rat) }\end{array}$ & $\begin{array}{l}\mathrm{LGS} \rightarrow \text { MG IA EPSP } \\
\text { peak amplitude } \\
(\mathrm{mV})\end{array}$ & $\begin{array}{l}\text { Rheobase } \\
\text { current }(\mathrm{nA})\end{array}$ & $\begin{array}{l}\text { AHP half-decay } \\
\text { time (ms) }\end{array}$ \\
\hline Normal & 4 & $50(9-22)$ & $1.38 \pm 0.14$ & $11.84 \pm 0.81$ & $11.15 \pm 0.32$ \\
\hline $3 \mathrm{~d}$ after LGS nerve crush & 4 & $61(10-21)$ & $2.86 \pm 0.22^{*}$ & $9.80 \pm 0.66$ & $11.29 \pm 0.14$ \\
\hline \multicolumn{6}{|l|}{ LGS crush + daily stim proximal tibial nerve } \\
\hline 1 h stim on days $1-3$ & 4 & $64(15-18)$ & $1.61 \pm 0.10$ & $9.25 \pm 0.86$ & $11.14 \pm 0.18$ \\
\hline 1 h stim on day 2 only & 2 & $29(14-15)$ & $2.36 \pm 0.07^{*}$ & $9.30 \pm 1.50$ & $11.59 \pm 0.45$ \\
\hline LGS crush + daily stim distal tibial nerve & 4 & $43(7-16)$ & $2.76 \pm 0.12^{*}$ & $7.48 \pm 0.45^{*}$ & $12.65 \pm 0.33$ \\
\hline $\begin{array}{l}\text { LGS crush + daily stim proximal tibial nerve } \\
\text { during lidocaine block }\end{array}$ & 4 & $40(8-13)$ & $1.52 \pm 0.09$ & $8.23 \pm 0.81$ & $12.73 \pm 0.41$ \\
\hline
\end{tabular}

Values are mean of means from individual animals \pm SEM. ${ }^{*} p<0.01$ for comparisons with normal group tested by nested ANOVA, Tukey's HSD post hoc test (see diagrams for treatment groups in Figs. 3 and 5 ).

Cope, 1998). Also, there is no evidence, and it seems unlikely, that MN properties would be altered over this short time by $1 \mathrm{~h}$ per day of electrical stimulation. Thus, the absence (with the one exception of rheobase current only in the group with distal tibial nerve stimulation) of statistically detectable differences in group means for rheobase current and AHP half-decay time in Table 1 suggests that there was no MN sampling bias contributing to differences in group means for IA EPSP amplitude.

\section{Synaptic enhancement blocked by axonal activity occurring outside the spinal cord}

The findings presented above did not specify where daily stimulation initiated its effect. Stimulation may have acted within the CNS by restoring impulse invasion to the central synapses made by crushed IA afferents (see Introduction). If this were the mechanism, then daily stimulation should be ineffective (i.e., should not prevent crush-induced enlargement of IA-EPSPs) when the evoked APs are blocked from propagating into the spinal cord. Results of this test are shown in Figure 5. Records in Figure $5 A$ taken in separate studies of four rats show that lidocaine was effective in blocking APs evoked in the periphery from propagating into the dorsal roots and that the block was reversible within minutes after washoff. Figure $5 B$ shows the surprising result that lidocaine block (Fig. $5 \mathrm{Bb}$ ) did not diminish the effect of proximal stimulation, which was equally effective in eliminating IA EPSP enlargement whether or not the evoked APs were blocked from reaching the spinal cord (see also Table 1). Evidence presented in the previous section suggested that that stimulation acted on the central portion of the crushed LGS nerve to prevent IA EPSP enlargement; findings presented in this section confine the effect of stimulation to the LGS nerve in the lower extremity (between the crush site near the LGS muscles and the lidocaine application site in the hip) (Fig. 5Bb).

\section{Signal for synaptic enhancement moves centrally by axonal transport}

We reasoned that the injury-induced signal for synaptic enhancement, if not carried centrally by AP activity, might be transmitted centrally by axonal transport. To test this possibility, axonal transport was blocked by application of colchicine central to the site of peripheral nerve crush. The effectiveness of colchicine blockade was tested by Western blot analysis for accumulation of the vesicular protein SV2 around the site of colchicine application. Accurate analysis per animal required more tissue than was obtainable from the LGS nerve alone, so colchicine was applied to the entire tibial nerve. Figure $6 \mathrm{~A}$ shows the SV2 accumulation produced by colchicine treatment of a tibial nerve in one rat. SV2 accumulation was attributed to colchicine and not to other aspects of the

\section{A}

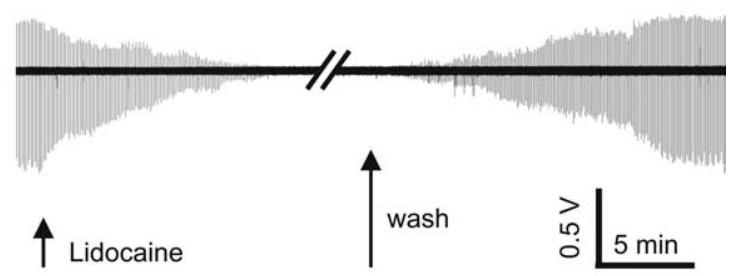

B

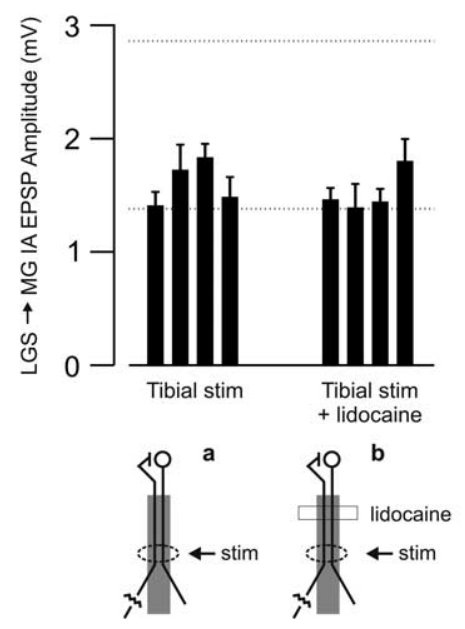

Figure 5. Daily tibial nerve stimulation acts outside spinal cord to prevent injury-induced synaptic enhancement. $A$, Compound APs in dorsal roots evoked by tibial nerve stimulation were completely blocked soon after applying lidocaine solution to the sciatic nerve (see $\boldsymbol{B} \boldsymbol{b}$ ) and restored within minutes of removing lidocaine and washing the nerve with saline (total block lasted $60 \mathrm{~min}$; diagonal lines truncate record). These results were reproduced in separate experiments on four rats to demonstrate the effectiveness of lidocaine in preventing APs evoked by tibial stimulation from propagating into the spinal cord. $B$, Bar graph shows mean \pm SEM for amplitude of LGS $\rightarrow$ MG IA EPSPs for each of four rats in two experimental groups distinguished by features illustrated in the associated diagrams (compare Fig. 3). Both groups had $3 \mathrm{~d}$ LGS nerve crush (jagged line in diagram) with daily stimulation (stim) of the tibial nerve (gray bar) at a proximal site (dotted oval and arrow) including axons from the crushed LGS nerve $(\boldsymbol{a}, \boldsymbol{b})$. In one group $(\boldsymbol{b})$, lidocaine was applied just before and washed off just after electrical stimulation to block central propagation of stimulus-evoked APs. Top and bottom horizontal dotted lines represent, respectively, the mean of mean values for LGS $\rightarrow$ MG IA EPSP amplitudes from rats with LGS nerve either crushed or normal. The graph shows that even with lidocaine block, tibial nerve stimulation eliminated the injury-induced increase in LGS $\rightarrow$ MG IA EPSP amplitude.

treatment procedure, because little SV2 accumulation was observed for the contralateral tibial nerve that received sham (saline) treatment. This analysis verified blockade of axon transport in all colchicine-treated rats from which IA EPSPs 

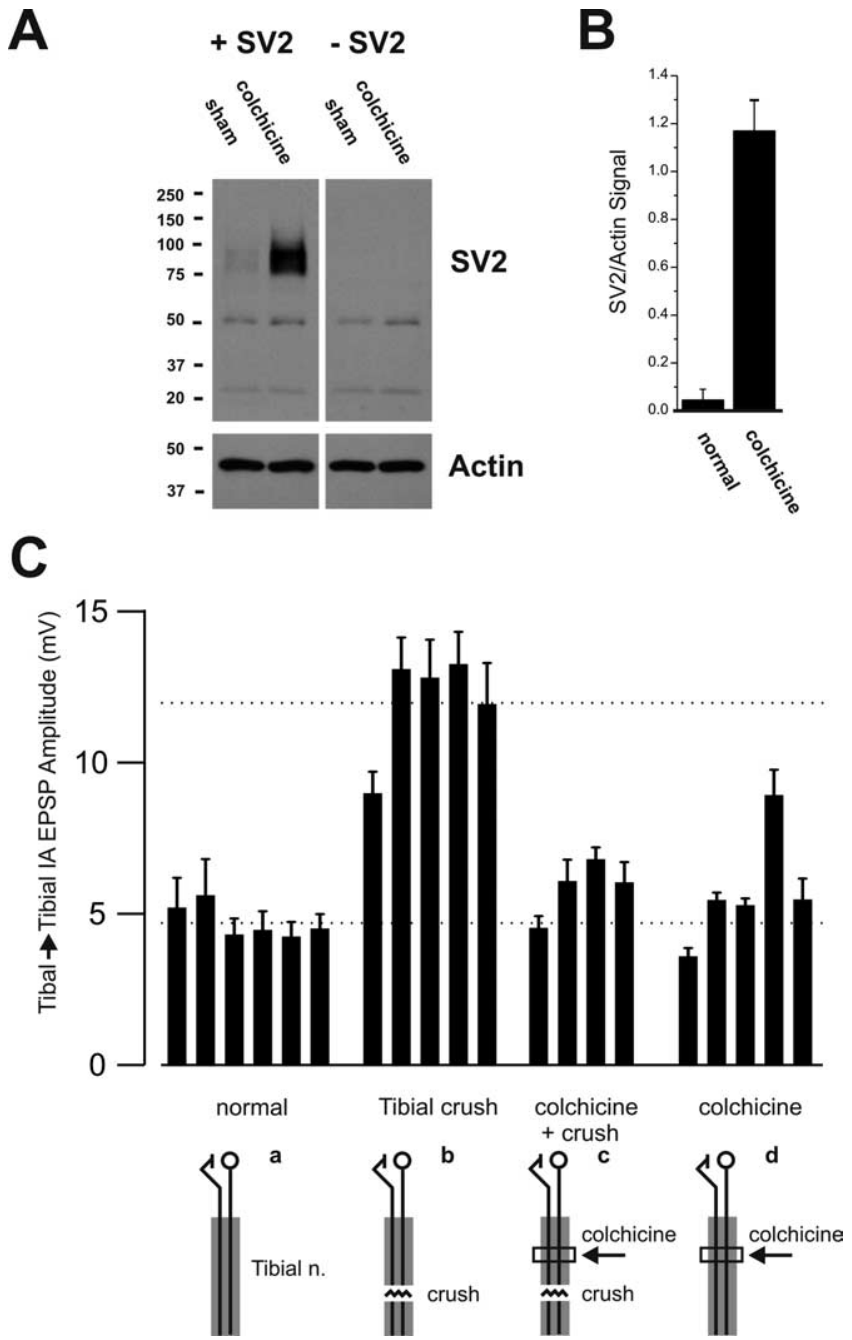

Figure 6. Acute colchicine treatment was effective in blocking axonal transport and in eliminating crush-induced IA EPSP enlargement. $A$, Western blots of SV2 [with (+SV2) or without ( - SV2) primary antibody] and actin for tibial nerve homogenates taken from sites of saline (sham) or colchicine application, respectively, on right and left tibial nerves from one rat. B, SV2 signal normalized to actin signal for right untreated control and left colchicine-treated tibial nerves. Bars and brackets represent mean $\pm S E M$, respectively, in arbitrary units of light intensity for nine rats from which IA EPSPs were recorded. C, Blocking axon transport eliminated injury-induced synaptic enhancement. The bar graph shows mean \pm SEM amplitude for tibial $\rightarrow$ tibial IA EPSPs sampled from individual rats in each of four experimental groups. Diagrams (compare Fig. 3) distinguish treatments (crush and/or colchicine) of the tibial nerve (Tibial n.; gray bar containing black lines representing tibial IA afferents and MNs) in relation to the IA-MN synapses at which EPSPs were recorded. Groups from left to right are as follows: $\boldsymbol{a}$, normal; $\boldsymbol{b}, 3 \mathrm{~d}$ tibial nerve crush (jagged line in diagram); $\boldsymbol{c}, 3 \mathrm{~d}$ tibial nerve crush preceded $1 \mathrm{~d}$ by acute colchicine treatment (rectangle and arrow); $\boldsymbol{d}$, intact tibial nerve with acute colchicine treatment. Top and bottom dotted lines represent, respectively, the mean of mean values for tibial $\rightarrow$ tibial IA EPSP amplitudes from rats with tibial nerve crushed and normal (see also Table 2).

were recorded (Fig. 6B). From these data, we inferred that colchicine treatment achieved transport blockade in at least some axons of the tibial nerve. Because we could not be certain that transport was blocked in all axons, including LGS axons, further study of IA EPSPs focused on the tibial nerve as a whole.

Figure $6 C$ shows that IA EPSPs evoked in tibial MNs were larger than normal in all rats studied $3 \mathrm{~d}$ after tibial nerve crush. The increase was statistically significant and amounted to values $\sim 2.5$ times larger than for normal rats (Table 2). In addition to demonstrating that the effects of crush generalize to IA-MN synapses in previously unstudied motor pools, this finding enabled us to test our hypothesis that the IA EPSP enlargement is prevented by blocking axon transport. Results presented in Figure $6 C$ and in Table 2 support this hypothesis, showing that the IA EPSP enlargement caused by nerve crush was eliminated by colchicine treatment (Fig. $6 \mathrm{Cc}$ ). To assess the possibility that colchicine treatment might mask the effect of nerve crush through a separate mechanism, the intact tibial nerves in some rats were treated with colchicine alone (Fig. 6Cd).

Figure 6 and Table 2 demonstrate that colchicine treatment of intact tibial nerves did not decrease or have any significant effect on IA EPSP amplitude. The conspicuously high mean for one rat with colchicine treatment of the intact tibial nerve (Fig. $6 C d$ ) may have resulted from unintended axonal injury caused at the time of the treatment, but this effect would contribute to and not mask enlargement caused by nerve crush.

Modification of nerve-crush enlargement of IA EPSPs depended on the relative timing of colchicine application. Crushinduced enlargement was eliminated as just described when colchicine was applied $1 \mathrm{~d}$ before nerve crush. In contrast, enlargement was not eliminated when colchicine treatment was applied in three additional rats at the same time as nerve crush ( $8.77 \pm 0.34 \mathrm{mV} ; n=33 ; p=0.043 \mathrm{vs}$ normal in Table 2$)$. This time dependence assists interpretation of the temporal development of the signal initiating IA EPSP enlargement.

Table 2 presents postsynaptic properties for many of the tibial MNs in which IA EPSPs were recorded. For AHP half-decay time, none of the treatment groups exhibited a significant difference from normal. This finding is consistent with previous demonstrations that changes in AHP occur more slowly after various nerve treatments in rat, including crush (Gardiner and Seburn, 1997). It also suggests that comparison of group means for IA EPSP amplitude was not confounded by bias in the MNs sampled in each group. Rheobase current for all treatment groups was significantly lower than normal as expected for MNs with crushed axons (Nakanishi et al., 2005). Interestingly, rheobase current was also significantly lower than normal after colchicine treatment of intact tibial nerves, and this effect may be relevant to signaling involved in regulating MN excitability (Nakanishi et al., 2005).

\section{Discussion}

The purpose of this study was to investigate the signaling mechanism(s) that triggers the increase in transmission strength in vivo at spinal synapses made by injured presynaptic neurons. The results were surprising in that the signal for initiating enhanced transmission strength at IA-MN synapses did not depend on altered synaptic activity, as expected. Synaptic enhancement was instead attributable to a molecular signal transported centrally by damaged primary sensory neurons from the peripheral site of axonal injury. Our findings provide the most direct evidence to date that a molecular injury signal acts in vivo and independent of synaptic activity to produce rapid and selective enhancement of synaptic strength in the mammalian CNS. The results were also novel in suggesting that the injury signal, although not dependent on the activity level of IA synapses, was modifiable by activity evoked in IA axons near the site of peripheral nerve crush.

\section{Independence from synaptic activity}

Our observations suggest that IA EPSP enlargement is initiated independently of changes in presynaptic activity at IA synapses. Colchicine blocked enlargement without preventing the injury 
Table 2. Nerve treatment effects on IA EPSP amplitude and other properties recorded from tibial MNs

\begin{tabular}{|c|c|c|c|c|c|}
\hline Treatment & $\begin{array}{l}\text { Number of } \\
\text { rats }\end{array}$ & $\begin{array}{l}\text { Number of MNs } \\
\text { (range MN/rat) }\end{array}$ & $\begin{array}{l}\text { Tibial } \rightarrow \text { tibial IA EPSP } \\
\text { peak amplitude (mV) }\end{array}$ & $\begin{array}{l}\text { Rheobase current } \\
(\mathrm{nA})\end{array}$ & $\begin{array}{l}\text { AHP half-decay } \\
\text { time (ms) }\end{array}$ \\
\hline Normal & 6 & $90(5-23)$ & $4.69 \pm 0.23$ & $9.86 \pm 0.59(76)$ & $17.09 \pm 1.38(76)$ \\
\hline Tibial nerve crush & 5 & $75(13-20)$ & $11.98 \pm 0.79^{*}$ & $7.15 \pm 0.71^{*}(64)$ & $17.26 \pm 1.97(62)$ \\
\hline Tibial nerve crush + colchicine & 4 & $66(10-22)$ & $5.81 \pm 0.48$ & $6.57 \pm 0.91^{*}(51)$ & $19.63 \pm 2.37(53)$ \\
\hline Tibial nerve intact + colchicine & 5 & $110(14-34)$ & $5.76 \pm 1.12$ & $6.92 \pm 0.50^{*}(89)$ & $17.28 \pm 0.57(58)$ \\
\hline
\end{tabular}

Values are mean of means from individual rats \pm SEM. * $p<0.01$ versus normal tested by nested ANOVA, Tukey's HSD post hoc test (see diagrams for treatment groups in Fig. 6).

discharge that occurs in response to crush and did not block central propagation of APs or impulse-evoked IA synaptic transmission (see Materials and Methods). In addition, the daily electrical stimulation that also prevented EPSP enlargement did not depend on propagation of the evoked APs into the spinal cord. This independence of IA EPSP enlargement from synaptic activity contrasts with the central sensitization associated with pain caused by nerve injury or inflammation, which is dependent on altered synaptic activity of primary afferents (Woolf and Costigan, 1999; Ji and Strichartz, 2004).

Peripheral nerve injury can also change postsynaptic activity, which is implicated in synaptic plasticity in spinal dorsal horn neurons (Flor et al., 2006). This postsynaptic mechanism might have influenced our results because the activity of MG MNs is known to increase soon after cutting the LGS nerve in cat (Pearson et al., 1999). If, however, IA EPSP enlargement depended on an injury-induced change in MN activity, then colchicine, which had no apparent effect on MN activity (see Materials and Methods) should not have prevented enlargement as it did. Thus, altered postsynaptic activity is not a good candidate signal for IA EPSP enlargement after nerve crush.

\section{"Disuse" hypothesis revisited}

Relative to well established rules by which synaptic strength is regulated by activity, Kuno's hypothesis that synapses are strengthened by presynaptic disuse (Kuno, 1995) is unique (see Introduction) and therefore important to examine. Central to this hypothesis were observations that IA EPSPs were enlarged under conditions, either nerve crush or chronic AP blockade that reduced or eliminated presynaptic activity. Despite an apparent association, there has been no definitive evidence that presynaptic inactivity is the primary signal for synaptic enhancement, and there are now significant challenges to the notion of a causal relationship. Data presented here show that IA EPSP enlargement after nerve crush can be uncoupled from synaptic activity (reversing enlargement was achieved in ways that did not rely on manipulating impulse-evoked transmission). Also, recent evidence shows that implantation of nerve cuffs, part of the procedure used to block AP propagation by tetrodotoxin infusion, induces local inflammatory responses and cytokine expression (Vince et al., 2005; Thil et al., 2007) that may trigger synaptic changes (see below). Thus, the potential generation of molecular signals from axons damaged by nerve cuffs confounds assigning synaptic activity as the primary trigger for IA EPSP enlargement. There is indeed little, if any, unequivocal evidence that a persistent change in steady-state transmission at IA-MN synapses can be initiated by a change in impulse activity at these synapses.

\section{Signals initiating synaptic enhancement}

If not presynaptic or postsynaptic activity, then what injury signal does trigger synaptic enhancement? The question can be addressed by distinguishing signals generated when axon damage interrupts central (retrograde) transport of constitutive mole- cules (negative injury signals) versus ones that are newly introduced, activated, or modified by axon injury (positive injury signals) (Ambron and Walters, 1996; Perlson et al., 2004; McMahon et al., 2005). Both negative and positive injury signals have been shown to initiate synaptic change (Lessmann, 1998; Mendell et al., 1999; Woolf and Costigan, 1999; Mendell and Arvanian, 2002; Kohno et al., 2003; Du and Poo, 2004; Sung et al., 2004; McMahon et al., 2005). For IA-MN synapses, deprivation of muscle-derived neurotrophin NT3 may contribute to the decline in EPSP amplitude that occurs $>1$ week after nerve section (Mendell et al., 2001). However, the enlargement that occurs earlier after nerve crush is probably not initiated by a negative injury signal, because we found that blocking axon transport in intact nerves had no discernable short-term effect on IA-MN synaptic function. We suggest instead that this early synaptic enhancement is initiated by a positive injury signal, because colchicine treatment as well as daily electrical stimulation had their synaptic effects only after nerve crush. All considered, it appears that nerve crush induces dynamic, time-dependent expression of both positive and negative signals (Mendell and Arvanian, 2002).

Definitive molecular identification of a positive injury signal has not yet been achieved for any system (Perlson et al., 2004). Results of the present study assist identification by establishing six criteria that must be met by the injury signal and/or nearby downstream mechanisms that initiate IA EPSP enlargement. First, the signal is carried by axon transport. Second, the signal is generated at or near the crush site, because synaptic enhancement is prevented by blocking axon transport $1 \mathrm{~cm}$ rostral to the crush. Third, the signal is generated within $1 \mathrm{~d}$ after crush, because colchicine prevents IA EPSP enlargement when applied $1 \mathrm{~d}$ before but not at the same time as nerve crush, meaning that the injury signal develops more quickly than the colchicine block. Fourth, the positive injury signal is either exclusively generated or selectively enabled by crushed IA axons, because IA EPSP enlargement is restricted to IA afferents that are crushed. Fifth, the signal does not depend on altered synaptic activity, but sixth, it is blocked or made otherwise ineffective by electrical stimulation of IA axons central to the crush but outside the CNS. In summary, our findings suggest that synaptic enhancement is triggered by a positive injury signal that develops quickly at the site of crush, moves centrally by axon transport, and acts selectively on synapses of crushed IA afferents, independent of IA-MN synaptic activity but dependent on IA axon firing.

The positive injury signal for IA-MN synaptic enhancement exists within an extensive and growing list of cellular and molecular changes elicited by nerve injury from neural and non-neural cells (e.g., Schwann cells and macrophages) to cause posttranslational modification and/or synthesis of axonal proteins (Ji and Strichartz, 2004; Jimenez et al., 2005; Navarro et al., 2007; Raivich and Makwana, 2007). Examination of potential signaling mechanisms underlying neural responses to injury has focused on neurotrophins and cytokines (Huang and Reichardt, 2003; Raivich and Makwana, 2007; Viviani et al., 2007). These and other can- 
didate signals can be tested for their in vivo relevance to IA-MN synaptic enhancement by applying criteria that we establish here. For example, the pro-inflammatory cytokine tumor necrosis factor $\alpha(\mathrm{TNF} \alpha)$ increases quickly at the site of peripheral nerve injury (Taskinen et al., 2000) and can increase glutamatergic synaptic strength (Bains and Oliet, 2007). However, TNF $\alpha$ does not qualify given evidence that it is not carried by retrograde transport to the DRG in injured nerves (Schafers et al., 2002) and is not restricted to primary afferents that are injured (Schafers et al., 2003). In contrast, the neurotrophin BDNF is a viable candidate by our criteria. BDNF increases at the injury site soon after axonal injury (Murphy et al., 1995; Omura et al., 2005) and in large DRG (IA) cell bodies of crushed primary axons (Cho et al., 1998; Michael et al., 1999); it is transported centrally when bound to its TrkB receptor, although the exact transport mechanism remains uncertain (Ginty and Segal, 2002). Also, BDNF trafficking can be influenced by neuronal activity (Nagappan and Lu, 2005), and if it were somehow impeded by stimulating axons electrically, then BDNF would meet all of our criteria. Another candidate signal worthy of careful consideration is the large, localized, and transient increase in free intracellular $\mathrm{Ca}^{2+}$ concentration occurring in the central tip of cut axons (Ziv and Spira, 1997). This $\mathrm{Ca}^{2+}$ signal drives protein kinase pathways (Ji and Strichartz, 2004) with the capacity to exert retrograde effects on synaptic transmission (Wang et al., 2005). For example, protein kinase $G$ is a positive retrograde injury signal that increases neuronal excitability (Sung et al., 2004, 2006) and has the potential to meet our criteria.

\section{Potential functional consequences}

These findings verify that mature IA-MN synapses have a large capacity for varying transmission strength in vivo (Kuno, 1995; Cope et al., 2001); the doubling of synaptic strength falls in the range manifested as LTP at other synapses (Holmes and Grover, 2006). In the specific case of peripheral nerve crush, muscle denervation precludes expression of reflex changes caused by IA EPSP enlargement; however, the positive injury signal suggested in the present study could initiate synaptic and sensorimotor changes if activated when the nerve remains intact, as, for example, during chronic inflammation. Enhanced IA transmission at synapses with MNs would cause stretch-reflex hyperexcitability and possibly spasticity (Nielsen et al., 2007), and at synapses with ascending pathways, if it occurs, might alter proprioception (Flor et al., 2006). Further assessment of functional relevance will rely on identifying the molecular signal and its activation mechanism.

\section{References}

Ambron RT, Walters ET (1996) Priming events and retrograde injury signals. A new perspective on the cellular and molecular biology of nerve regeneration. Mol Neurobiol 13:61-79.

Bains JS, Oliet SH (2007) Glia: they make your memories stick! Trends Neurosci 30:417-424.

Burke RE, Rymer WZ, Walsh JV (1978) Relative strength of synaptic input from short-latency pathways to motor units of defined type in cat medial gastrocnemius. J Neurophysiol 39:447-458.

Cho HJ, Kim JK, Park HC, Kim JK, Kim DS, Ha SO, Hong HS (1998) Changes in brain-derived neurotrophic factor immunoreactivity in rat dorsal root ganglia, spinal cord, and gracile nuclei following cut or crush injuries. Exp Neurol 154:224-230.

Cope TC, Seburn K, Buck CR (2001) How does nerve injury strengthen Ia-motoneuron synapses? In: Motor neurobiology of the spinal cord (Cope TC, ed), pp 271-304. Washington, DC: CRC.

Davis GW (2006) Homeostatic control of neural activity: from phenomenology to molecular design. Annu Rev Neurosci 29:307-323.

Du JL, Poo MM (2004) Rapid BDNF-induced retrograde synaptic modification in a developing retinotectal system. Nature 429:878-883.
Flor H, Nikolajsen L, Staehelin Jensen T (2006) Phantom limb pain: a case of maladaptive CNS plasticity? Nat Rev Neurosci 7:873-881.

Gallego R, Kuno M, Nunez R, Snider WD (1979) Disuse enhances synaptic efficacy in spinal mononeurones. J Physiol (Lond) 291:191-205.

Gardiner PF, Seburn KL (1997) The effects of tetrodotoxin-induced muscle paralysis on the physiological properties of muscle units and their innervating motoneurons in rat. J Physiol (Lond) 499:207-216.

Ginty DD, Segal RA (2002) Retrograde neurotrophin signaling: Trk-ing along the axon. Curr Opin Neurobiol 12:268-274.

Haftel VK, Bichler EK, Nichols TR, Pinter MJ, Cope TC (2004) Movement reduces the dynamic response of muscle spindle afferents and motoneuron synaptic potentials in rat. J Neurophysiol 91:2164-2171.

Haftel VK, Bichler EK, Wang QB, Prather JF, Pinter MJ, Cope TC (2005) Central suppression of regenerated proprioceptive afferents. J Neurosci 25:4733-4742.

Holmes WR, Grover LM (2006) Quantifying the magnitude of changes in synaptic level parameters with long-term potentiation. J Neurophysiol 96:1478-1491.

Huang EJ, Reichardt LF (2003) Trk receptors: roles in neuronal signal transduction. Annu Rev Biochem 72:609-642.

Jackson P, Diamond J (1977) Colchicine block of cholinesterase transport in rabbit sensory nerves without interference with the long-term viability of the axons. Brain Res 130:579-584.

Ji RR, Strichartz G (2004) Cell signaling and the genesis of neuropathic pain. Sci STKE 2004:reE14.

Jimenez CR, Stam FJ, Li KW, Gouwenberg Y, Hornshaw MP, De Winter F, Verhaagen J, Smit AB (2005) Proteomics of the injured rat sciatic nerve reveals protein expression dynamics during regeneration. Mol Cell Proteomics 4:120-132.

Kohno T, Moore KA, Baba H, Woolf CJ (2003) Peripheral nerve injury alters excitatory synaptic transmission in lamina II of the rat dorsal horn. J Physiol (Lond) 548:131-138.

Kuno M (1995) The synapse: function, plasticity, and neurotrophism. Oxford: Oxford UP.

Lessmann V (1998) Neurotrophin-dependent modulation of glutamatergic synaptic transmission in the mammalian CNS. Gen Pharmacol 31:667-674.

Lev-Tov A, Pinter MJ, Burke RE (1983) Posttetanic potentiation of group Ia EPSPs: possible mechanisms for differential distribution among medial gastrocnemius motoneurons. J Neurophysiol 50:379-398.

Li JY, Volknandt W, Dahlstrom A, Herrmann C, Blasi J, Das B, Zimmermann H (1999) Axonal transport of ribonucleoprotein particles (vaults). Neuroscience 91:1055-1065.

Manabe T, Kaneko S, Kuno M (1989) Disuse-induced enhancement of Ia synaptic transmission in spinal motoneurons of the rat. J Neurosci 9:2455-2461.

McMahon SB, Cafferty WB, Marchand F (2005) Immune and glial cell factors as pain mediators and modulators. Exp Neurol 192:444-462.

Mendell L, Johnson R, Munson J (1999) Neurotrophin modulation of the monosynaptic reflex after peripheral nerve transection. J Neurosci 19:3162-3170.

Mendell LM, Arvanian VL (2002) Diversity of neurotrophin action in the postnatal spinal cord. Brain Res Brain Res Rev 40:230-239.

Mendell LM, Munson JB, Arvanian VL (2001) Neurotrophins and synaptic plasticity in the mammalian spinal cord. J Physiol (Lond) 533:91-97.

Michael GJ, Averill S, Shortland PJ, Yan Q, Priestly JV (1999) Axotomy results in major changes in BDNF expression by dorsal root ganglion cells: $\mathrm{BDNF}$ expression in large trkB and trkC cells, in pericellular baskets, and in projections to deep dorsal horn and dorsal column nuclei. Eur J Neurosci 11:3539-3551.

Michaelis M, Liu X, Janig W (2000) Axotomized and intact muscle afferents but no skin afferents develop ongoing discharges of dorsal root ganglion origin after peripheral nerve lesion. J Neurosci 20:2742-2748.

Miyata Y, Yasuda H (1988) Enhancement of Ia synaptic transmission following muscle nerve section: dependence upon protein synthesis. Neurosci Res 5:338-346.

Murphy PG, Grondin J, Altares M, Richardson PM (1995) Induction of interleukin-6 in axotomized sensory neurons. J Neurosci 15:5130-5138.

Nagappan G, Lu B (2005) Activity-dependent modulation of the BDNF receptor TrkB: mechanisms and implications. Trends Neurosci 28:464-471.

Nakanishi ST, Cope TC, Rich MM, Carrasco DI, Pinter MJ (2005) Regula- 
tion of motoneuron excitability via motor endplate acetylcholine receptor activation. J Neurosci 25:2226-2232.

Navarro X, Vivo M, Valero-Cabre A (2007) Neural plasticity after peripheral nerve injury and regeneration. Prog Neurobiol 82:163-201.

Nielsen JB, Crone C, Hultborn H (2007) The spinal pathophysiology of spasticity-from a basic science point of view. Acta Physiol (Oxf) 189:171-180.

Omura T, Sano M, Omura K, Hasegawa T, Doi M, Sawada T, Nagano A (2005) Different expressions of BDNF, NT3, and NT4 in muscle and nerve after various types of peripheral nerve injuries. J Peripher Nerv Syst 10:293-300.

Pearson KG, Fouad K, Misiaszek JE (1999) Adaptive changes in motor activity associated with functional recovery following muscle denervation in walking cats. J Neurophysiol 82:370-381.

Perlson E, Hanz S, Medzihradszky KF, Burlingame AL, Fainzilber M (2004) From snails to sciatic nerve: retrograde injury signaling from axon to soma in lesioned neurons. J Neurobiol 58:287-294.

Raivich G, Makwana M (2007) The making of successful axonal regeneration: genes, molecules and signal transduction pathways. Brain Res Rev $53: 287-311$

Schafers M, Geis C, Brors D, Yaksh TL, Sommer C (2002) Anterograde transport of tumor necrosis factor- $\alpha$ in the intact and injured rat sciatic nerve. J Neurosci 22:536-545.

Schafers M, Geis C, Svensson CI, Luo ZD, Sommer C (2003) Selective increase of tumour necrosis factor-alpha in injured and spared myelinated primary afferents after chronic constrictive injury of rat sciatic nerve. Eur J Neurosci 17:791-804.

Seburn KL, Cope TC (1998) Short-term afferent axotomy increases both strength and depression at Ia-motoneuron synapses in rat. J Neurosci 18:1142-1147.

Stent GS (1973) A physiological mechanism for Hebb's postulate of learning. Proc Natl Acad Sci USA 70:997-1001.
Sung YJ, Walters ET, Ambron RT (2004) A neuronal isoform of protein kinase $\mathrm{G}$ couples mitogen-activated protein kinase nuclear import to axotomy-induced long-term hyperexcitability in Aplysia sensory neurons. J Neurosci 24:7583-7595.

Sung YJ, Chiu DT, Ambron RT (2006) Activation and retrograde transport of protein kinase $\mathrm{G}$ in rat nociceptive neurons after nerve injury and inflammation. Neuroscience 141:697-709.

Taskinen HS, Olsson T, Bucht A, Khademi M, Svelander L, Roytta M (2000) Peripheral nerve injury induces endoneurial expression of IFN-gamma, IL-10 and TNF-alpha mRNA. J Neuroimmunol 102:17-25.

Thil MA, Duy DT, Colin IM, Delbeke J (2007) Time course of tissue remodelling and electrophysiology in the rat sciatic nerve after spiral cuff electrode implantation. J Neuroimmunol 185:103-114.

Turrigiano G, Nelson S (1998) Thinking globally, acting locally: AMPA receptor rurnover and synaptic strength. Neuron 21:933-941.

Vince V, Thil MA, Gerard AC, Veraart C, Delbeke J, Colin IM (2005) Cuff electrode implantation around the sciatic nerve is associated with an upregulation of TNF-alpha and TGF-beta 1. J Neuroimmunol 159:75-86.

Viviani B, Gardoni F, Marinovich M (2007) Cytokines and neuronal ion channels in health and disease. Int Rev Neurobiol 82:247-263.

Wang JQ, Arora A, Yang L, Parelkar NK, Zhang G, Liu X, Choe ES, Mao L (2005) Phosphorylation of AMPA receptors: mechanisms and synaptic plasticity. Mol Neurobiol 32:237-249.

Webb CB, Cope TC (1992) Modulation of la EPSP amplitude: the effects of chronic synaptic inactivity. J Neurosci 12:338-344.

Woolf CJ, Costigan M (1999) Transcriptional and posttranslational plasticity and the generation of inflammatory pain. Proc Natl Acad Sci USA 96:7723-7730.

Ziv NE, Spira ME (1997) Localized and transient elevations of intracellular $\mathrm{Ca}^{2+}$ induce the dedifferentiation of axonal segments into growth cones. J Neurosci 17:3568-3579. 